\title{
Diagnosis of Human Papillomatosis by Polymerase Chain Reaction in Cases of Divergence Between Results of Hybrid Capture and Papanicolaou Cytology
}

\author{
Luiz Carlos Garcez Novaes ${ }^{1}$, \\ Maria Rita Carvalho Garbi Novaes ${ }^{2}$ \\ and Augusto Simões-Barbosa ${ }^{1}$
}

\author{
${ }^{1}$ Catholic University of Brasília, ${ }^{2}$ School of Meical Health; \\ Brasilia, DH, Brazil
}

\begin{abstract}
As various types of human papillomavirus (HPV) are involved in the pathogenesis of cervical cancer, correct diagnosis is of fundamental importance for screening programs. We evaluated the divergence of results between Papanicolaou cytology and hybrid capture by PCR detection of HPV DNA . A transversal study was conducted on 70 women attending private gynecological clinics in Brasilia, Brazil. PCRs were conducted with specific primers for general and high-risk HPV DNA. Based on the PCR results, hybrid capture was a superior diagnostic technique. When Papanicolaou was compared with the molecular biology methods, it was found that a positive Papanicolaou result does not necessarily indicate the presence of HPV. The agreement between PCR and hybrid capture results can be attributed to the fact that both methods detect latent infection, while Papanicolaou detects only microscopic cellular alterations.
\end{abstract} Key Words: HPV, diagnosis, hybrid capture, PCR.

It is widely accepted that genital human papillomavirus (HPV) is the main cause of cervical intraepithelial neoplasia and carcinoma [1,2]. In Brasilia, the capital of Brazil, the routine methods for the diagnosis of this infection and its lesions are oncologic cytology, by traditional Papanicolaou cytology (PC), the standard exam, and Hybrid Capture ${ }^{\circledR}(\mathrm{HC})$ technique and its variant HC II. The latter is able to detect the DNA of this virus; indirectly, it infers the risk of evolution of cervical lesions to cancer, by classifying viral types into high-risk (HR) and low-risk (LR) HPV.

The classic clinical recommendation for the investigation of HPV by HC is the finding of atypical squamous cells of undetermined significance (ASC-US) in PC, which means no evolution to cancer, based on cytological aspects. $\mathrm{HC}$ is quickly becoming popular, and solicitation of the HC exam by gynecologists is now very common. The finding of an HR HPV requires a prompt decision by the physician, while an LR HPV will need more frequent out-patient exams and long-term observation [3]. The detection of HR HPV by a molecular biology diagnostic test can facilitate diagnosis, avoiding the risk of pathological evolution and the need for sequential PC exams [4].

The magnitude of preventive exams for the detection of uterine cervix cancer may be assessed by considering that every woman, from the time she first becomes sexually active, should be submitted to a PC or an equivalent exam. The initial routine recommendation is one exam per year; after three

Received on 27 December 2005; revised 17 April 2006.

Address for correspondence: Dr.Catholic University of Brasília, Campus II - Pós-graduação em Biotecnologia Genômica, SGAN 916, módulo B, Asa Norte. Zip code: 70790-160. Brasília DF, Brazil. Phone/Fax: 61347 4797. E-mail: sbarbosa@pos.ucb.br

The Brazilian Journal of Infectious Diseases 2006;10(3):169-172. (C) 2006 by The Brazilian Journal of Infectious Diseases and Contexto Publishing. All rights reserved. consecutive negative results, there should be one exam every three years for the rest of a woman's life, as long as the results remain negative, according to World Health Organization WHO recommendations [5,6]. In Brazil, it has been estimated that 35 million PC exams are done every year, about 800,000 in Brasília alone, according to the Health Department of the Capital of Brazil (1999). The HC exam is not a routine procedure in the public health centers in Brasilia, generally being solicited by physicians in private gynecology clinics, due to the fact that such an exam is about 50 to 100 times more expensive than conventional PC.

There can be divergence between $\mathrm{PC}$ and $\mathrm{HC}$; the sensibility and specificity of neither method is $100 \%$ accurate. The specificity for $\mathrm{PC}$ is about 60 to $75 \%$ according to a comparative study using gold-standard histopathology [7]. On the other hand, HC presents sensitivity and specificity of up to $93 \%$ and $61 \%$, respectively [8]. Since adequate examination, prognostic evaluation and treatment are directly dependent on the definition of infection status, discordance in HPV diagnostic results creates a dilemma for physicians. Whenever $\mathrm{PC}$ and $\mathrm{HC}$ results are in discordance, diagnosis of HPV by polymerase chain reaction (PCR) can provide a third option $[9,10]$. Although more time-consuming, PCR can provide consistent information for a clinical decision. We used PCR to look for HPV DNA in patient samples that had divergent results reported by $\mathrm{PC}$ versus $\mathrm{HC}$; we also examined possible causes and consequences of the divergent results by investigating the inherent limitations of the various techniques.

\section{Material and Methods}

A transversal study was made of 70 women attending gynecology clinics at private health centers in Brasilia, Brazil, from November 2003 to July 2004. The age of the patients varied from 22 to 73 years old (average, 43 years). This 
population was randomized in terms of behavior and clinical signs and symptoms. Following recommendations by the national committee of medical research ethics, all patients signed an informed consent form. The criterion for inclusion of the patients in the study group was to present contemporary but divergent results between $\mathrm{PC}$ and $\mathrm{HC}$, within a maximum period of 30 days. The $\mathrm{HC}$ and $\mathrm{PC}$ exams were processed in different specialized laboratories in Brasilia in order to counteract individual subjective aspects as well as specific variations of results. The divergent exam results were grouped (Table 1).

Cervical samples were collected from the patients by gynecologists with a sterile cervix brush. The samples were immediately placed in 600 microliter sterile microtubes containing preserving solution. The samples were kept at $4^{\circ} \mathrm{C}$ up to 48 hours before DNA extraction. The DNA was obtained using Version E of the Easy-DNA ${ }^{\mathrm{TM}} \mathrm{Kit}$, as recommended (Invitrogen, USA). Qualitative and quantitative analysis of the DNA samples were performed by electrophoresis in Trisborate-EDTA buffer and ethidium bromide stained agarose gels, as described elsewhere [11]. For quality control, DNA extraction and PCR reactions were done in separate rooms, with different sets of micropipettes. From 50 to $100 \mathrm{ng}$ DNA was used for each DNA amplification reaction. Routinely, a negative control was performed by omitting DNA from the

Table 1. Description of divergent results between hybrid capture and Papanicolaou cytology for human papillomavirus diagnosis

\section{Case Description}

1 Positive $\mathrm{HC}$ for every risk (HR and/or LR) and normal PC.

2 Positive $\mathrm{HC}$ for LR and PC results for CIN I(LSIL), CIN II (HSIL), CIN III, CIS or SCCA.

3 Negative $\mathrm{HC}$ and PC results for ASC-US or ASG-US.

4 Negative HC and PC results for CIN I(LSIL), CIN II (HSIL), CIN III, CIS or SCCA.

HC - hybrid capture; PC - Papanicolaou cytology; ASC-US - atypical squamous cells of undetermined significance; ASG-US - atypical glandular cells of undetermined significance; CIN - cervical intraepithelial neoplasia; LSIL - low-grade cervical intraepithelial neoplasia; HSIL high-grade cervical intraepithelial neoplasia; CIS - carcinoma in situ; SCCA - squamous cell carcinoma. reaction. All cervicovaginal swab samples were first tested by PCR with a set of primers for amplification of a human housekeeping gene, glyceraldheyde-3-phosphatedehydrogenase (GAPDH), as previously described by Lobo et al. [12]. Amplification with these primers indicated the absence of inhibitors and adequate quality of the DNA samples for PCR assays.

The BIOPAP - Detection Kit ${ }^{\circledR}$ was employed, as recommended (Biotools B\&M Labs, Madrid, Spain) in order to detect HPV DNA by PCR and to determine HR and LR virus type. Briefly, the standardized procedure for PCR amplification for HPV included 5 minutes of predenaturation at $94^{\circ} \mathrm{C}$, followed by 35 cycles, each consisting of 30 seconds of denaturation at $94^{\circ} \mathrm{C}, 60$ seconds of annealing at $55^{\circ} \mathrm{C}$, and 60 seconds of extension at $72^{\circ} \mathrm{C}$, with a 10 -minute final postextension step. The reagents were adjusted to a final volume of $25 \mu \mathrm{L}$. The $450 \mathrm{bp}$ PCR product represents the universal HPV DNA amplicon, and the $250 \mathrm{bp}$ PCR product indicates the presence of HR-type HPV. Consequently, LR-type HPV is indicated to be present when the first PCR product is detected but not the second one. In addition, each sample was tested twice by independent PCR assays. No conflicting or doubtful results were seen throughout the screening of the samples. Along with the positive control in the Biotools kit, DNA from HeLa and SeHa cells were routinely included as positive controls. Statistical agreement among diagnostic results and their correlations were calculated by the Fisher test, after translation of qualitative to quantitative data. Translation was based on the conversion table of PC results of the Cervical Intraepithelial Neoplasia (CIN) system to the Bethesda Cytology System for cervicovaginal smears. Finally, the probability of significance $(p=0.05)$ was determined by the Chi-square test.

\section{Results}

Figure 1 illustrates a set of PCR results from cervicovaginal DNA samples of patients in this study group. All samples were initially tested with primers for amplification of a human DNA segment corresponding to GAPDH and gave a single amplification product of $783 \mathrm{bp}$, indicating that they had adequate quality for PCR assays (Figure $1 \mathrm{~B}$ ). Using specific HPV primers to, three possibilities were envisaged: 1) no

Table 2. Comparative results of PCR versus Papanicolaou cytology and PCR versus hybrid capture for HPV diagnosis

Concordance

\begin{tabular}{|c|c|c|c|c|c|}
\hline \multirow[t]{2}{*}{ Cases } & \multicolumn{2}{|c|}{ Agreement } & \multicolumn{2}{|c|}{ Disagreement } & \multirow[t]{2}{*}{ Cases } \\
\hline & PCR/PC & PCR/HC & PCR/PC & PCR/HC & \\
\hline$+/+$ & $9(36 \%)$ & $20(55.6 \%)$ & $26(57.8 \%)$ & $18(52.9 \%)$ & $+/-$ \\
\hline$-/-$ & $16(64 \%)$ & $16(44.4 \%)$ & $19(42.2 \%)$ & $16(47.1 \%)$ & $-/+$ \\
\hline Total & $25 / 70$ & $36 / 70$ & $45 / 70$ & $34 / 70$ & Total \\
\hline
\end{tabular}

PCR - Polymerase Chain Reaction; PC - Papanicolaou cytology; HC - Hybrid capture; +/+ or -/-, both exams present positive or negative results, respectively; $+/-$, the first exam is positive while the second is negative; $-/+$, the first exam is negative while the second is positive. 
Figure 1. Results of polymerase chain reaction tests for detection of HPV infection in cervicovaginal DNA samples from patients presenting contemporary but divergent results of Papanicolaou cytology and hybrid capture. A - PCR with GAPDH primers. B - PCR with HPV specific primers from BIOPAP detection kit (Biotools); 1 to 10 - cervicovaginal DNA samples from 10 different patients; $\mathrm{N}$ - negative control; $\mathrm{P}$ - positive control using the Biotools kit. The arrowheads indicate the molecular weight of the DNA fragments.
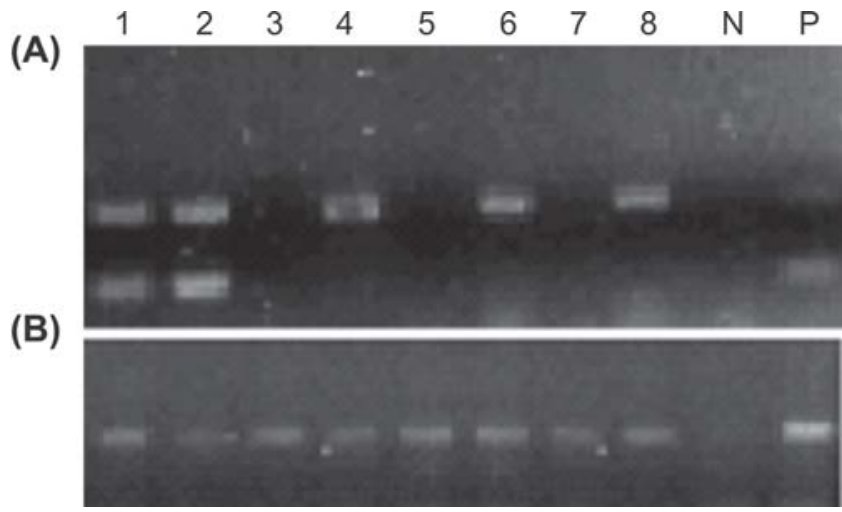

products amplified, 2) two amplification products of 450bp and 250bp, and 3) a single amplification product of $450 \mathrm{bp}$ (Figure $1 \mathrm{~A}$ ). Number one means absence of HPV infection (samples 1, 2, 5, 7 and 9). The second case means presence of HPV infection of HR-type (samples 3 and 4). In the third case, although HPV is confirmed by the presence of the universal fragment of $450 \mathrm{bp}$, the infection is due to the LR-type, since the HR-type is excluded by the lack of the $250 \mathrm{bp}$ amplicon (samples 6, 8 and 10).

Table 2 summarizes the comparative diagnostic results and illustrates the distribution of positive and negative cases in terms of concordance for PCR/PC and PCR/HC. Although there were 25 cases of agreement for PCR/PC and 36 cases for PCR/ $\mathrm{HC}$, there were also 45 cases of disagreement for PCR/PC and 34 for PCR/HC. Concordance with PCR results was higher for $\mathrm{HC}$. There was a significant negative coincidence between the results from PC versus PCR ( $p=0.002844$, Chi-square test). $\mathrm{HC}$ and PCR, on the other hand, gave similar results ( $\mathrm{p}=$ 0.014429 , Chi-square test). In addition, when only the positivity or negativity of results were compared for detection of HRHPV and LR-HPV by PCR and HC, the results for these two types of tests were strongly and positively associated ( $\mathrm{p}=$ $1.15 \times 10^{-18}, \chi^{2}$ test).

\section{Discussion}

Chronic HPV infection in the uterine cervix causes morphocytological changes in cells. HR-HPV can cause highgrade lesions and cancer; however, LR-HPV lesions do not evolve to cancer $[13,14]$. Therefore, HPV diagnosis at early stages of the infection is of fundamental importance. A correct diagnosis will help reduce the incidence of cervix cancer and its complications. Previous studies have demonstrated that the Papanicolaou test is not efficient and adequate when used as the sole method for diagnosis of HPV infection [15]. In fact, if a molecular-biology-based method is not employed to specifically distinguish subtypes of HPV, it will be necessary to repeat Papanicolaou exams several times during follow-up in order to decide on clinical versus surgical treatment.

Molecular methods have been extensively used for detection and typing of HPV [7]. Hybrid Capture ${ }^{\circledR}$ (HC) technology detects nucleic acid targets directly by immunological reaction after hybridization with RNA probes, the precision of which has proven to be comparable to target amplification methods by PCR $[16,17]$. The use of a molecular technique determines the real status of the infection and enables distinction among pathotype strains in order to develop specific treatment strategies. However, the Papanicolaou test is still the most widely-used methodology for primary screening of cellular abnormalities and some genital infections in most developing countries, since it is a simple and low-cost, widely-available technique. However, there is a need for a more sensitive and specific low-cost method. Till such methods become available, epidemiological investigation with these improved molecular detection methods can help reveal the necessity for adequate diagnosis and treatment of the infected population, dispensing unnecessary aggressive treatment and providing information for surveillance, healthcare policy and health-care management programs.

We used PCR to detect HPV DNA in order examine divergent results of $\mathrm{PC}$ versus $\mathrm{HC}$ diagnostic procedures. The rate of misdiagnosis, false-positives and false-negatives, found in these two latter methods is high enough to characterize a real problem, though with uncertain proportions in a largescale analysis. PCR was able to detect a larger number of positives than $\mathrm{HC}$ and many wrongly-diagnosed samples based on PC, indicating higher sensitivity and sufficient clinical specificity. Nevertheless, PCR results do not guarantee absence of discrepancy. Various explanations for divergence of PC, HC and PCR results are possible. The variability of results in a Papanicolaou exam can be affected by individual factors of the patient, by the experience of the examiner, and by the subjectivity of the examination itself. PCR and HC, both molecular biology techniques, are standardized and may be compared among different laboratories and research centers $[18,19]$.

The inclusion criterion was divergence between the PC and $\mathrm{HC}$ diagnostic tests. We found that divergence was greater between PCR and PC than between PCR and HC. Some divergence was expected, based on previous studies [20]. PCR and HC frequently gave results different from PC. This fact shows that a positive $\mathrm{PC}$ result will not necessarily indicate the presence of HPV. The opposite is also true. In other words, the virus may be present even if morphological modifications in cells of the uterine cervix are not identified in a PC exam. In 
fact, we found more cases of false-negatives (26/45) than falsepositives (19/45) by PC, which could be due to latent infections with HPV. On the other hand, when comparing only positivity or negativity between PCR and HC, the result from one exam (positive or negative) was significantly associated with the same result by the other exam, different from what was found in a previous study in Brazil [19]. In cases of HR- and LR-HPV genotyping, PCR and HC were very concordant $\left(\mathrm{p}=1.15 \times 10^{-18}\right)$, as expected when these two strategies are employed [20].

It has been previously shown that PCR has a sensitivity of 50\% (47/94) and $37.2 \%(35 / 94)$ in comparison with PC and $\mathrm{HC}$, respectively. The findings indicate more chances of falsenegative than false-positive misdiagnosis by the $\mathrm{HC}$. Moreover, the accordance between PCR and HC was strongly decreased in cases of ASC-US, compared to other grade lesions [18]. Although quantitative $\mathrm{HC}$ data was not available for our study, we hypothesized that the cut-off values for $\mathrm{HC}$ may be a cause of misdiagnosis. It is becoming evident that a PCR-based technology is a better choice methodology for the detection and genotyping of HPV infection. Our results suggest that the concordance between PCR and HC results and their high sensitivity can be attributed to the fact that both methods detect latent infection, while PC is dependent on the subjective appearance of cellular alterations visualized through microscopy. PCR is a high specific and sensitive diagnosis tool for the detection of HPV DNA, and it could be indicated for tracking HPV of cervicovaginal smears. However, due to the complexity of these techniques, the necessity for specific material and sophisticated equipment, the costs of $\mathrm{PC}$ and $\mathrm{HC}$ exams are still comparatively high.

\section{Acknowledgments}

The authors thank their colleagues Doctors Evandro Silva, Maria Quitéria Santos, Jackson Pontes and Valquíria Primo for collecting samples and Prof. Dr. Eduardo Leonardecz for statistical assistance. This work was supported by the Program on Genomic Science and Biotechnology of the Catholic University of Brasilia.

\section{References}

1. Walboomers J.M.M., Jacobs M.V., Manos M.M., et al. Human Papillomavirus is a necessary cause of invasive cervical cancer world-wide. J Pathol 1999;189:12-9.

2. Schiffman M.H., Castle P. Epidemiologic studies of a necessary causal risk factor: human papillomavirus infection and cervical neoplasia. J Natl Cancer Inst 2003;95:E2.
3. O'Meara A.T. Present standards for cervical cancer screening. Curr Opin Oncol 2002;14:505-11.

4. Lorincz A.T. Molecular methods for the detection of human Papillomavirus infection. Obstet Gynecol Clin North Am 1996;23:707-30.

5. Sellors J.W., Karwalajtys T.L., Kaczorowski J., et al. Incidence, clearance and predictors of human Papillomavirus infection in women. CMAJ 2003;168:421-5.

6. World Health Organization. An overview of selected curable sexually transmitted disease. Global Program on AIDS report. Geneva: World Health Organization, 1995.

7. Hubbard R.A. Human papillomavirus testing methods. Arch Pathol Lab Med 2003;127:940-5.

8. Dillner J. Primary screening for human Papillomavirus infection. Best Pract Res Clin Obstet Gynaecol 2001; $15: 743-57$.

9. Renshaw A.A. Measuring sensitivity in gynecologic cytology: a review. Cancer 2002;96:210-7.

10. Lorincz A.T, Hildesheim A., Herrero R., et al. Comparison of hybrid capture II HPV DNA detection and conventional PAP smear for cervical cancer screening in Costa Rica. $17^{\text {th }}$ International Papillomavirus Conference, 1999.

11. Sambrook J., Fritsch E.F., Maniatis T. New York, NY. Molecular cloning. A laboratory manual. Cold-Spring Harbor, 2001.

12. Lobo T., Feijo G., Carvalho S., et al. A Comparative Evaluation of the Papanicolaou Test for the Diagnosis of Trichomoniasis. Sex Transm Dis 2003;9:694-9.

13. Pfister J. WSLH Summary: Epidemiology \& Public Health. Cervical Cancer Screening Program Public Health, 2002.

14. Laimins L.A. The biology of human papillomaviruses: from warts to cancer. Infect Agents Dis 1993;2:74-86.

15. Koss L.G. The Papanicolaou test for cervical cancer detection. A triumph and a tragedy. JAMA 1989;261:737-43.

16. Castle P., Lorincz A., Mielzynska-Lohnas I., et al. Results of Human Papillomavirus DNA testing with the Hybrid Capture 2 are reproducible. JCM 2002;40:1088-90.

17. Clavel C., Masure M., Putaud Thomas K. Hybrid Capture II, a new sensitive test for Human Papillomavirus detection comparison with hybrid capture I and PCR results in cervical lesions. J Clin Pathol 1998;51:737-40.

18. Zazove P., Reed B., Gregoire L., et al. Low false-negative rate of PCR analysis for detecting human papillomavirus-related cervical lesions. JCM 1998;36:2708-13.

19. Nonnenmacher B., Breitenbach V., Villa L.L., et al. Genital Human Papillomavirus infection identification by molecular biology among asymptomatic women. Rev Saude Publica 2002;36:95-100.

20. Peyton C.L., Schiffman M., Lorincz A.T., et al. Comparison of PCR and Hybrid Capture based Human Papillomavirus detection system using multiple cervical specimen collection strategies. JCM 1998;36:3248-54. 